\title{
New IJH guidelines highlight greater transparency
}

\author{
Douglas Sipp • Toshio Suda
}

Published online: 6 April 2011

(c) The Japanese Society of Hematology 2011

Biomedical research has grown to become a truly international enterprise in which scientific advances are increasingly linked to financial, legal, social, and ethical issues of deep import. They have an impact not only on the conduct of experiments in laboratories or trials in clinical settings but importantly on the manner in which the results of such research are communicated as well. Such communications are a fundamental responsibility of all scientists, serving to push back the frontiers of human knowledge, promote better understanding and application of new discoveries, and accelerate discussion among peers and the general public. Indeed, it is no exaggeration to say that the dissemination of findings is fundamental to the success and sustainability of the scientific enterprise.

At the International Journal of Hematology, we take the responsibilities associated with our role as stewards over the reporting of hematological research very seriously. It has always been the policy of the journal to coordinate the review of submitted manuscripts in a timely, fair, and constructive manner and to support authors in their efforts to gain the widest possible audience for their work. As part of this commitment, the editors have developed a set of guidelines to assist them in the process of creating manuscripts with an emphasis on quality, clarity, accessibility, and impact.

We now feel, however, that it is time to revise these guidelines to bring them up to date with emerging global standards for the ethical publication of biomedical results and to make explicit a number of expectations that were tacit in the previous versions of the IJH "Information for Authors". These changes are in general conformity with the recommendations set forth in the Uniform Requirements for Manuscripts (URM) formulated by the International Council of Medical Journal Editors (ICMJE), which were developed "to help authors and editors in their mutual task of creating and distributing accurate, clear, and easily accessible reports of biomedical studies", and are available from the ICMJE website (http://www.icmje.org). Our newly revised "Information for Authors" document can be downloaded from the journal website at http://www. springer.com/medicine/internal/journal/12185.

The main additions to the "Information for Authors" can be found in the expanded Editorial Policies section, which now outlines expectations for specific areas, such as the conduct of animal studies, authorship, reporting of potential conflicts of interest, and research on human subjects. These changes to our authors' guide are by no means radical departures from the high ethical standards we believe are maintained in the great majority of laboratories and clinical research programs. We have made them explicit, however, in line with the growing international expectations for greater transparency, accountability, and disclosure on the part of scientific researchers.

The new requirements for the disclosure of author affiliations or activities that could conceivably lead to conflicts of interest serve as a case in point. It is no longer safe to rely on the assumption that financial or other relationships on the part of authors will go unquestioned when scientific results are challenged. We have therefore followed the ICMJE in requiring that all authors accompany research submissions with a simple statement, in the form of a checklist, that describe any financial or personal relationships that might be perceived as potentially biasing the reporting of their findings. This is not to say that bias inevitably results from such relationships, only that their disclosure is an essential component in ensuring transparency and impartiality.

The definition of authorship is a second area in which the new guidelines are more explicit. We require that all authors on an article's byline "have made substantive 
contributions to the experimental design, the acquisition and/or analysis of research data, or the preparation of the manuscript, and must agree to accept responsibility for the aspects of the work with which they were involved". Specifically, this excludes the practices of "gift authorship", in which author status is conferred on someone, generally in the same department or laboratory, who has not made a substantive contribution to the work described, and "guest authorship", in which an eminent researcher or other authority is made an author without having participated in the research. The converse situation of "ghost authorship", in which professional writers are engaged to craft a manuscript without being listed in the byline, is also prohibited.

A third area in which the "Information for Authors" has been expanded is in the requirement for the registration of prospective clinical trials prior before or by the time of subject enrollment. We agree with the principles outlined by the ICMJE in calling for the mandatory registration of such research as a safeguard against the selective reporting of trial results. Accordingly, the IJH will now only accept reports of results from clinical trials that have been entered in a freely accessible, not-for-profit public registry, such that details of the design, methodology, and outcome measures prior to their inception.

We encourage all prospective authors to read the revised guidelines published at http://www.springer.com/medicine/ internal/journal/12185, and to comply with all requirements and recommendations when submitting their manuscripts, in the interests of prompt and uninterrupted editorial review. We welcome feedback from the hematology community regarding these new measures, which we feel will further strengthen the research quality and public trust in our field.

\section{Managing Editor}

Douglas Sipp

Editor-in-Chief

Toshio Suda, M.D. 